Hy-Ol-Ne diagram it becomes clear that there is no systematic tendency for the non-rise samples to be more alkaline. Furthermore, when the Niggli Quartz Index for each sample - a measure of the amount of silica relative to $\mathrm{Al}, \mathrm{Fe}$, $\mathrm{Mg}, \mathrm{Na}$ and $\mathrm{K}$ in the rock-is plotted as a function of distance from the rise it becomes equally apparent that there is no significant tendency for the non-rise basalts to have lower silica saturations. In short, as far as these key parameters are concerned the basalts are indistinguishable. Nor do any differences become apparent when the data are plotted on a silica-alkali diagram of the type used by MacDonald and Katsura (J. Petrol., 5, 82; 1964) to differentiate between alkaline and tholeiitic basalts in Hawaii.

Bonatti and Fisher interpret this information to mean that both rise and non-rise basalts must be produced within the same depth range, and hence the same pressure range, within the upper mantle. This then implies that the differences in heat flow between the rise region and other areas do not indicate that beneath the rise there is a higher thermal zone which reaches down to the region of magma generation. The heat source which accounts for the higher heat flow must lie above the region of magma production, though it is not immediately clear whether or not such a conclusion conflicts with the simple picture of mantle upwelling at ridges and sinking beneath trenches. An alternative interpretation-that pockets of magma produced beneath the rise are caught in, and spread with, the lower crust only to break through to the surface at a later date--seems to be ruled out by the fact that during the spreading the magma would undergo fractionation and differentiation, processes which would themselves give rise to a compositional difference between rise and non-rise basalts.

\section{PROTEINS \\ Finding the Way}

from our Molecular Biology Correspondent ARGUably the most interesting problem in protein chemistry at present is just how a randomly coiled polypeptide chain finds its way through the almost infinite maze of possible conformational states that it may assume to the haven of the native conformation. It can safely be asserted only that the chain cannot within any reasonable time scale approach it by a process of random wandering, but that there must be pathways determined by the sequence. A widely favoured hypothesis is that the starting points are segments that readily form an ordered structure, such as a few turns of $\alpha$-helix or a $\beta$-fold. This would imply that the parts of the sequence most prone to enter these conformations in the random chain will also be $\alpha$-helical in the finished article -the folded, rigid globular protein. Scheraga has given arguments, based on the helix-forming potential of particular amino-acids, compared with their occurrence in $\alpha$-helixes in proteins of known structure, that such is indeed the case. Not even the most sanguine supporters of the theory would claim, however, that the evidence is conclusive, but at present there is no other to which its opponents can rally.

Scheraga and his colleagues are now attempting to place the calculations of helix stability in relation to amino-acid composition on a quantitative basis, and their strategy is outlined in a new series of articles. The stability of an $\alpha$-helix is defined by two equilibrium constants, $\sigma$ and $s$, which define respectively the initiation of an $\alpha$-helix in a randomly coiled homopolymer chain, and the incorporation of a residue into an existing helix. The well known cooperativity of helix formation and breakdown carries the implication that $\sigma$ is much the smaller. In homopolymers helix-coil transition profiles can be used to determine both parameths. Von Dreele et al. (Macromolecules, 4, $396 ; 1971$ ) have re-worked the statistical mechanics of the system, with some new approximations, to include polymers containing a proportion of a foreign residue with different $\sigma$ and $s$. From the effect of such "guest" residues on the helix-coil transition profile of the "host" polymer, values of the constants can be determined, and Von Dreele et al. (ibid., 408) report that for a test case the $\sigma$ and $s$ values extracted for a given "guest" residue agree well with those determined

\title{
Arvin as an Anticoagulant
}

Fibrinogen is a plasma protein which plays a constitutive and initiative part in the haemostatic mechanism of wound healing. But it is also involved in the formation in vivo of fibrin deposits with the grave clinical consequences of disseminated intravascular coagulation, for example, thrombosis and arteriosclerosis. The production of thrombin in vivo triggers the following simplified scheme:

$$
\begin{aligned}
& \text { Fibrinogen } \\
& (\mathrm{A} \alpha, \mathrm{B} \beta, \gamma)_{2} \underset{\mathrm{Ca}^{++}}{\stackrel{\text { Thrombin }}{\longrightarrow}} \\
& \text { Fibrin clot } \\
& (\alpha, \beta, \gamma)_{p} \\
& \underset{\mathrm{Ca}^{++}}{\stackrel{\text { Factor XIII }}{\longrightarrow}} \\
& \left(\alpha^{p}, \beta, \gamma^{2}\right)_{p}
\end{aligned}
$$

where $p$ denotes polymer and factor XIII $\equiv$ fibrin stabilizing factor (FSF).

In recent years it has been discovered that certain enzymes from snake venoms can induce formation of fibrin clots from plasma fibrinogen and these clots differ in stability and structure from thrombininitiated clots. The most used venom enzymes, arvin ('Ancrod') and reptilase, remove only fibrinopeptide $A$, whereas thrombin removes fibrinopeptides $\mathrm{A}$ and $B$ from the $A \alpha$ and $B \beta$ chains of the fibrinogen molecule respectively. Further differences in the mechanisms of these enzymes are described by Mattock and Esnouf in next Wednesday's Nature New Biology. They use the technique of SDSacrylamide gel electrophoresis to explain at a molecular level the action of arvin as an anticoagulant and thus help to bridge the gap which exists between empirical clinical practice and biochemical rationale.

Mattock and Esnouf corroborate the findings of McKee et al. (Proc. US Nat. Acad. Sci., 66, 738; 1970) that thrombin in the presence of calcium ions activates factor XIII which mediates clot stabilization by the formation of $\gamma$ chain dimers and $\alpha$ chain complexes of molecular weight in excess of 500,000 . They show that reptilase works in a similar manner but in the absence of calcium ions and this would explain its role as a potent topical haemostatic agent. On the other hand, arvin is shown to remove 31,000 molecular weight portions of the $\alpha$ chain with no formation of $\alpha$ chain complexes. It can be suggested that the arvin susceptible areas of the $\alpha$ chains are those which would have taken part in amide cross-

linking mediated by factor XIII. Arvin was also shown not to activate factor XIII because very little $\gamma$ dimers were formed. Non-crosslinked fibrin clots are readily degraded by intrinsic plasmin to soluble non-clottable fibrin degradation products and the role of arvin as an anticoagulant by a defibrination mechanism was demonstrated at a subunit level.

It is interesting to speculate whether arvin might be thrombolytic because Mattock and Esnouf indicate that it degrades the $\alpha$ chain complexes produced by thrombin. Their argument for suggesting that this highly interesting proteolytic activity of arvin could not result from an additional plasmin-like enzyme in the arvin preparation is not supported by the recent evidence that plasmin produces 30,000 molecular weight fragments from the $A \alpha$ chain of fibrinogen (Gaffney and Dobos, FEBS Lett., 15, 13 ; 1971). But in spite of this contradiction, Mattock and Esnouf have amply demonstrated the mechanism by which an arvin preparation acts as a controlled anticoagulant and they illustrate the part that biochemical techniques can play in understanding the principles underlying thrombus formation and dissolution. 